\title{
Transatlantica
}

Revue d'études américaines. American Studies Journal

\section{Edgar Allan Poe et les meubles de la philosophie}

\section{Thomas Constantinesco}

\section{(2) OpenEdition}

Journals

Édition électronique

URL : https://journals.openedition.org/transatlantica/6370

DOI : $10.4000 /$ transatlantica.6370

ISSN : 1765-2766

Éditeur

Association française d'Etudes Américaines (AFEA)

Référence électronique

Thomas Constantinesco, «Edgar Allan Poe et les meubles de la philosophie», Transatlantica [En ligne], 1 | 2013, mis en ligne le 14 février 2014, consulté le 03 février 2023. URL : http://

journals.openedition.org/transatlantica/6370 ; DOI : https://doi.org/10.4000/transatlantica.6370

Ce document a été généré automatiquement le 3 février 2023.

\section{(c) (i) (9)}

Creative Commons - Attribution - Pas d'Utilisation Commerciale - Pas de Modification 4.0 International - CC BY-NC-ND 4.0

https://creativecommons.org/licenses/by-nc-nd/4.0/ 


\title{
Edgar Allan Poe et les meubles de la philosophie
}

\author{
Thomas Constantinesco
}

« Why has America never expressed itself philosophically?

Or has it - in the metaphysical riot of its greatest literature? ॥

(Cavell, 1992, 33)

1 De ces deux questions que Stanley Cavell pose à l'Amérique, mais aussi à la philosophie et à la littérature - à sa philosophie et sa littérature -, un premier constat se dégage en forme d'hypothèse : la philosophie ne serait pas chez elle en Amérique comme elle le serait, disons, en Grèce, en Allemagne ou même en France. Question de lieu, donc, d'appartenance, d'ancrage dans le territoire d'une nation que la pensée imagine en même temps qu'elle s'y établit, mais aussi d'installation dans une langue et une écriture. Ou plutôt, en l'occurrence, question de non-lieu, d'absence de lieu idoine d'où la philosophie pourrait parler en son propre nom et s'exprimer en tant que philosophie. L'Amérique, ou l'utopie de la philosophie. Sans domicile fixe une fois transplantée au Nouveau Monde ${ }^{1}$, la philosophie ne serait plus à sa place, elle n'aurait plus de place et devrait, pour se dire, trouver à s'établir ailleurs, en invitée ou en parasite, mais toujours en étrangère, investir d'autres lieux et d'autres discours, tenter de faire bon ménage avec d'autres modalités du savoir, d'autres pratiques de la pensée, et singulièrement la littérature. Or la littérature à laquelle songe ici Cavell - celle d'Emerson et de Thoreau d'abord, mais aussi de Hawthorne, Melville et Poe -, si elle paraît disposée à héberger la philosophie, voire à se mettre en ménage avec elle, semble peu désireuse de la traiter avec égards ou ménagements. D'un tempérament rebelle et même séditieux, prompte à se révolter contre l'autorité du logos, la littérature américaine promet à la philosophie de nombreuses scènes de ménage en lui offrant une hospitalité non dépourvue d'hostilité ${ }^{2}$. L'«insurrection métaphysique » en quoi consisterait le geste littéraire en Amérique est annonciatrice d'un joyeux remueménage à tous les sens du terme : la cohabitation de la littérature et de la philosophie sous le même toit et dans le même texte présage un désordre intérieur, un chaos 
domestique, qui pourrait bien s'étendre au-delà et venir agiter la communauté tout entière. Aux États-Unis plus qu'ailleurs peut-être, le partage entre les disciplines, et en particulier entre philosophie et littérature, qui s'établit avec les romantiques d'Iéna et que confirme symboliquement en 1800 l'ouvrage de Mme de Staël, De la littérature, aurait donc paradoxalement donné lieu au brouillage des frontières censées délimiter ces deux modes d'écriture et de pensée.

Qu'en est-il dès lors de ce ménage entre littérature et philosophie en Amérique ? Que signifie pour la philosophie habiter en littérature et pour la littérature être l'hôte de la philosophie? Comment être chez soi quand on a élu domicile chez un autre? À l'inverse, comment se sentir chez soi quand un étranger y séjourne? Et qu'est-ce que s'installer dans la maison d'autrui? Qu'est-ce qu'emménager? Avec quels meubles? Quels sont au juste les meubles de la philosophie? Y aurait-il une philosophie du meuble ? À cette série de questions, que me suggère celles que lance Stanley Cavell dans Senses of Walden en guise de programme philosophique et qui me servent ici de point de départ, je me propose de chercher quelques éléments de réponse dans un court texte d'Edgar Poe curieusement intitulé «The Philosophy of Furniture» et publié pour la première fois en mai 1840 dans Burton's Gentleman's Magazine ${ }^{3}$.

Sans doute Poe emploie-t-il ici le mot de " philosophy » dans le sens qu'il revêt jusqu'au $\mathrm{XVIII}^{\mathrm{e}}$ siècle, où il désigne encore la théorie générale d'un domaine de connaissances donné avant d'être progressivement remplacé dans cette acception par celui de "science ${ }^{4}$. Et sans doute envisage-t-il sa "philosophie de l'ameublement " (c'est ainsi que Baudelaire traduit le titre) avec autant d'ironie facétieuse qu'il exposera en 1846 une «philosophie de la composition » dans laquelle il énonce, on le sait, sa conception de la poésie comme un art de l'effet ${ }^{5}$ - mais Henri Justin nous a appris depuis longtemps que, si Poe "défie toute lecture naïvement sérieuse ", «le pendule critique est bien dans le champ de la raison moqueuse et joueuse, de la parodie lucide et critique " (Justin, 1991, 2 et 23) : le canular n'empêche pas qu'il y ait là un enjeu d'importance, il en serait plutôt le moyen. Assurément aussi, Poe joue avec la popularité du syntagme "The Philosophy of... » dans l'Amérique du premier XIX siècle, où il sert de titre quasi générique à de très nombreux ouvrages et conférences. Lui-même n'a-t-il pas publié des comptes rendus de The Philosophy of Human Life par un certain Amos Dean et de Sacred Philosophy of the Seasons par un dénommé Henry Duncan dans les livraisons de février et mars 1840 de Burton's, ainsi qu'un résumé de A Lecture on the Philosophy of Vegetation par un W. A. Seeley dans le numéro du 8 mars 1845 du Broadway Journal ? La formule est même tellement en vogue que Dickens, de retour de sa première tournée aux États-Unis, se moquera de cette prétention américaine à philosopher à tout va dans son Martin Chuzzlewit qui paraît en 1843 :

"What course of lectures are you attending now, ma'am?" said Martin's friend, turning again to Mrs Brick.

"The Philosophy of the Soul - on Wednesdays."

"On Mondays?"

"The Philosophy of Crime."

"On Fridays?"

"The Philosophy of Vegetables."

"You have forgotten Thursdays - the Philosophy of Government, my dear," observed the third lady.

"No," said Mrs Brick. "That's Tuesdays."

"So it is!" cried the lady. "The Philosophy of Matter on Thursdays, of course."

"You see, Mr Chuzzlewit, our ladies are fully employed," said Bevan. 
"Indeed you have reason to say so," answered Martin. "Between these very grave pursuits abroad, and family duties at home, their time must be pretty well engrossed." (Dickens, 2004, 284)

Le comique de répétition qui régit la scène suggère qu'à force de vouloir philosopher dans tous les sens, l'Amérique brasse surtout du vide. Les Américaines courent les conférences pour tuer le temps et dissimulent la vacuité de leurs existences à coup de fausse philosophie. Mais surtout, elles demeurent parfaitement insensibles à toute l'ironie de leurs réponses monotones, comme si aux États-Unis, on avait aussi peu d'esprit que cette pauvre Mrs Brick au patronyme si éloquent. Si en 1840 Poe pouvait discrètement tourner en dérision l'ambition de ses concitoyens en matière de philosophie tout en reprenant à son compte un titre déjà en passe d'être éculé, la satire de l'Amérique qu'offre le roman de Dickens explique en partie pourquoi, lorsqu'il décide en mai 1845 de republier "The Philosophy of Furniture " dans le Broadway Journal dont il est devenu rédacteur en chef, il choisit de l'intituler plus simplement «House Furniture ». C'est toutefois sous son titre original mais dans sa seconde leçon que le texte figure dans l'édition des œuvres complètes de Poe (Poe, 1978, 494-504). Peut-être est-ce en raison de ce titre marquant, une fois déconnecté d'un contexte qui pourrait en faire un objet de ridicule, que "The Philosophy of Furniture » a retenu l'attention de quelques grands lecteurs de Poe. Parmi eux Baudelaire, qui en propose une première traduction en octobre 1852, Benjamin, pour qui Poe est «le premier physiognomoniste de l'intérieur» (Benjamin, 1989, 41), ou encore Lacan, qui évoque "l'ambiance que l'auteur de la philosophie de l'ameublement sait faire surgir de notations presque impalpables » (Lacan, 1999, 35). Malgré son titre à la fois engageant et quelque peu mystérieux, ce texte a cependant peu intéressé les philosophes et la philosophie, et Mark Kingwell résume l'opinion de beaucoup quand il affirme sans ménagements dans "Tables, Chairs, and Other Machines for Thinking»: "["The Philosophy of Furniture"] is not by a philosopher, it is not really a philosophy, and it is not really about furniture. » (Kingwell, 2002, 234) Mais si Poe n'est pas un philosophe, il n'est pas sûr en revanche que l'on ne puisse pas lire dans sa "philosophie de l'ameublement » quelque chose du « remue-ménage métaphysique » dont parle Stanley Cavell et qui serait la marque de la relation paradoxale qu'entretiennent en Amérique littérature et philosophie. Telle est du moins la direction que je souhaiterais suivre dans cette étude.

\section{Du meuble en philosophie}

5 Avant d'ouvrir le texte plus avant, commençons par remarquer que les meubles jouissent en philosophie d'un statut doublement ambigu. D'une part, et en dépit d'une étymologie qui renvoie aux biens dits meubles, que l'on peut déplacer ou transporter avec soi, le mobilier suppose toujours un espace intérieur fixe et immobile dans lequel il prend place et dont il concourt à l'aménagement. À tout meuble, son immeuble. La question du meuble, de l'ameublement, en vient donc à connoter l'installation durable dans un lieu, l'occupation habituelle et continue d'un espace et, partant, une relative absence de mobilité. Établir sa résidence quelque part, c'est même, dans l'un des sens anciens du mot, demeurer chez soi, garder la chambre, ce qui laisse entendre la présence de quelque maladie qui ne tardera pas à emporter le résident qui en est affligé vers sa résidence dernière, comme si s'installer conduisait immanquablement à interrompre le mouvement de la vie, autant du reste que celui de la pensée. En effet, 
une longue tradition philosophique a fait sienne l'idée d'Aristote et des péripatéticiens, selon laquelle la philosophie est affaire de promenade (de peripatein, "se promener ») : on ne philosopherait bien qu'au dehors et en marchant ${ }^{6}$. Il suffit pour s'en convaincre d'accompagner dans ses rêveries le promeneur solitaire de Rousseau ou de se souvenir du mot de Nietzsche dans le Crépuscule des idoles : «Être cul-de-plomb, voilà, par excellence, le péché contre l'esprit! Seules les pensées que l'on a en marchant valent quelque chose.» (Nietzsche, 1974, 16) Avec ce trait, Nietzsche rappelle un autre "redoutable marcheur», Thoreau, qui, «dans l'ultime essai qu'il écrira, intitulé justement Marcher (Walking), [...] donne sur le mode héroï-comique ses quartiers de noblesse à la marche. » (Pétillon, 2000, 7). Et il n'est pas impossible qu'en l'écrivant, Nietzsche ait songé à Emerson dont il était un grand lecteur et qui lançait, dans son essai sur Montaigne paru dans Representative Men (1850): " The philosophy we want is one of fluxions and mobility. (Emerson, 1983, 696) Philosophie du mouvement et en mouvement, philosophie du dehors et des grands espaces : «the world is all outside: it has no inside", dit encore Emerson dans "Experience" (Emerson, 1983, 481). Le modèle du philosophe transcendantaliste, "The American Scholar », c'est le philosophe de plein air, celui dont la pensée se forge au contact de la nature, loin des bibliothèques et de leurs rayonnages poussiéreux ; c'est Thoreau gravissant le mont Ktaadn dans les forêts du Maine ou arpentant la bande de sable du cap Cod pour emmener la philosophie aussi bien dans les bois qu'à la plage ${ }^{7}$. Derrière cette apologie philosophique de l'extériorité et du mouvement transparaît également, en filigrane, une célébration de la vie contre la sclérose mortifère d'une pensée figée, confinée aux limites étouffantes d'un espace clos. Dans cette perspective, l'idée même d'une " philosophie de l'ameublement » ne peut qu'être entachée de soupçon, dans la mesure où elle entend ramener la pensée à l'intérieur du foyer et menace de l'immobiliser au service de l'économie domestique. C'est cependant méconnaître le paradoxe qui soustend cette tradition de la philosophie au grand air dont le transcendantalisme est l'avatar américain et qui, tout en affirmant le privilège de la déambulation et en faisant de la marche le lieu mobile de la pensée, passe sous silence la nécessité de s'asseoir à une table ou dans un fauteuil pour consigner les pensées venues à l'esprit pendant la marche et grâce à elle, et ainsi prolonger, voire achever, la réflexion philosophique entamée au dehors. Première ambiguïté du meuble, implicitement tenu pour une entrave à la philosophie et pourtant essentiel à la formation de la pensée. De ce point de vue, en consacrant quelques pages aux meubles et à leur philosophie, Poe anticipe la position d'un Flaubert, la cible de Nietzsche dans le fragment cité plus haut, pour qui " on ne peut penser et écrire qu'assis 8 .» Car la question des meubles de la philosophie, c'est aussi, en un sens, celle du lieu même où se fait la pensée (Kingwell, 2002, 231).

D'autre part, dans les rares moments où la philosophie se pique d'ameublement, les pièces de mobilier dont il est fait mention apparaissent dans leur généralité la plus grande, à titre d'exemples prototypiques, et leur destin semble être soit de s'effacer derrière l'Idée dont elles ne sont jamais que la représentation sensible dégradée, soit de s'évanouir dans le tourbillon du doute sceptique, ce qui revient finalement au même. Ainsi en va-t-il du lit que Socrate convoque au livre X de La République (596b-598b) pour exposer sa théorie des Formes : remontant de l'imitation de l'apparence d'un lit par le peintre, à l'imitation de l'idée du lit par l'ouvrier, à la production de l'essence du lit par Dieu, Socrate conclut que seul ce troisième lit est réel et vrai, car lui seul existe « dans la nature des choses ", remarquant au passage que Dieu n'est pas le simple «fabricant particulier d'un lit particulier », mais « réellement le créateur d'un lit réel » (Platon, 
1995, 361). Mais ce lit métaphysique est surtout un lit sur lequel il est impossible de se coucher, sinon en pensée, puisqu'il n'existe qu'en tant qu'idée. Escamoté derrière l'essence qui préside à sa fabrication, l'objet lit n'est mentionné dans sa matérialité sensible que pour être dépassé par sa forme intelligible (éidos) sur laquelle nous sommes invités à tourner l'œil de l'âme à défaut de pouvoir y reposer notre corps. De même chez Descartes, la méditation conduit le métaphysicien à douter de la réalité du monde extérieur, à commencer par la pièce dans laquelle il se trouve et les objets qui l'entourent, "par exemple, que je sois ici, assis auprès du feu, vêtu d'une robe de chambre, ayant ce papier entre les mains, et autres choses de cette nature " (Descartes, 1992, 59). Mais on notera qu'avant même d'être engloutis par le doute radical, les meubles cartésiens ont déjà disparu : s'il se décrit assis près du feu tenant à la main le récit de l'expérience de pensée qu'il est à la fois en train de faire et de composer, Descartes ne fait nulle mention du siège ni de la table nécessaires à sa mise en scène de leur effacement. Deuxième ambiguïté du meuble en philosophie dont la présence à la fois générique et fantomatique n'est jamais que le prélude à sa destruction par la pensée (Kingwell, 2002, 236). De ce point de vue également, Poe semble prendre le contre-pied de la tradition métaphysique occidentale en consacrant la seconde partie de sa " philosophie de l'ameublement » à la description patiente d' " une petite chambre sans prétention, dans la décoration de laquelle il n'y a rien à reprendre » (Poe, 1951, 976).

7 Par son seul titre, «The Philosophy of Furniture » traduirait donc ironiquement un double mouvement de rejet de la tradition philosophique, ce qui n'a rien de très surprenant quand on songe à l'hostilité souvent mordante de Poe à l'égard des grands noms de la spéculation métaphysique, dont il oppose les élucubrations et les égarements aux rigueurs de la raison rationnelle (Richard, 1974, 292-93). Ainsi, dans «Mellonta Tauta », Kant est joyeusement rebaptisé M. «Cant », tandis que dans « BonBon ", le diable explique tranquillement au personnage éponyme, restaurateur de son état et métaphysicien hors pair, comment son régime alimentaire se compose exclusivement de philosophes! (Poe, 1984a, 874 et 164-180)

8 Ce refus de la métaphysique, s'il est perceptible dans bien des contes, s'observe également de l'une à l'autre des deux versions du texte qui nous occupe. En effet, au moment de republier « The Philosophy of Furniture » dans le Broadway Journal, Poe non seulement efface du titre toute référence à la philosophie pour adopter celui, plus neutre, de "House Furniture », mais il retire également le premier paragraphe qui s'ouvrait sur un aphorisme apocryphe attribué à Hegel :

"Philosophy," says Hegel, "is utterly useless and fruitless, and, for this very reason, is the sublimest of all pursuits, the most deserving of our attention, and the most worthy of our zeal" - a somewhat Coleridegy assertion, with a rivulet of deep meaning in a meadow of words. It would be wasting time to disentangle the paradox - and the more so as no one will deny that Philosophy has its merits, and is applicable to an infinity of purposes. There is reason, it is said, in the roasting of eggs, and there is philosophy even in furniture - a philosophy nevertheless which seems to be more imperfectly understood by Americans than by any civilized nation upon the face of the earth'.

9 Il est probable que le changement d'intitulé ait entraîné la suppression de ce paragraphe et ce, pour des raisons assez évidentes de cohérence de la composition. Il n'en reste pas moins que ce geste vient redoubler la mise sous rature de la philosophie qu'opère de fait la leçon de 1845 dans laquelle le mot "philosophy " n'apparaît plus qu'une seule fois, au détour d'une phrase sur laquelle nous reviendrons et qui l'associe 
désormais aux motifs de l'erreur, de la faute de goût et de la perversion esthétique : " Glare is a leading error in the philosophy of American household decoration - an error easily recognized as deduced from the perversion of taste just specified ». Dès 1840 toutefois, la référence à la philosophie n'a rien d'une révérence. D'entrée de jeu, la pseudo-citation de Hegel, qualifiée de « jargon » dans les Marginalia de juin 1849 (Poe, 1984b, 1459), met à mal l'autorité du discours philosophique et suggère que la vérité dont il se prétend porteur pourrait bien n'être qu'un faux en écriture. Contrefaisant la voix de la philosophie, Poe en brouille à la fois l'origine, puisque la paternité de cette maxime pourrait revenir à Coleridge autant qu'à Hegel (voire à Tertullien si l'on suit les Marginalia), et la destination, dans la mesure où son sens méandreux se perd rapidement dans une prairie de mots. De surcroît, pour profonde que soit la signification de la formule, celle-ci paraît vouée à rester littéralement inter-dite, car le ruisseau qu'elle forme demeure distinct des mots au milieu desquels elle circule pourtant. Hors langage, la vérité de la philosophie semble à jamais inintelligible et ce serait perdre son temps que de chercher à en élucider les insondables paradoxes qui, au bout du compte, ne nous sont d'aucune utilité (Fleming, 2010, 581).

10 Cependant, en même temps que Poe renvoie Hegel et Coleridge dos à dos et tourne en dérision l'idéalisme européen, il affirme malicieusement l'universalité de l'effort philosophique qui peut, par nature, s'appliquer à tous les sujets, depuis la cuisson des œufs jusqu'à l'aménagement intérieur : «There is reason, it is said, in the roasting of eggs, and there is philosophy even in furniture ». Détournant un vers de Pope ("The Vulgar boil, the Learned roast an Egg » [Pope, 1963, 652]), il suggère implicitement que c'est peut-être là, dans la reconnaissance de la dignité philosophique des choses et des actes ordinaires, que se marque une singularité américaine en matière de philosophie, même si l'Amérique elle-même le méconnaît encore. Derrière la facétie, qui débouche une fois passé ce premier paragraphe sur une longue condamnation du mauvais goût affiché par les contemporains de Poe dans la décoration de leurs intérieurs, s'entend ainsi la revendication discrète d'une philosophie de l'ordinaire que ne renierait pas Emerson et qui n'a sans doute pas échappé à Cavell, puisqu'il rappelle dans « Being Odd, Getting Even (Descartes, Emerson, Poe) » tout l'intérêt de Poe pour les événements du quotidien (Cavell, 2003, 108-09).

11 Associant de manière quelque peu surprenante Emerson et Poe, Cavell voit en particulier dans «Self-Reliance » et "The Imp of the Perverse» une entreprise parallèle de relecture critique, sinon de Descartes, au moins du lien entre la pensée et l'existence qui se noue dans l'énoncé du cogito. Dans l'un et l'autre cas, il y va pour Cavell de la tonalité d'une écriture, de la manière dont les voix de la philosophie résonnent dans les textes de la littérature américaine du XIX ${ }^{e}$ siècle. De Poe, il dit notamment ceci :

The sound of Poe's prose, of its incessant and perverse brilliance, is uncannily like the sound of philosophy as established in Descartes, as if Poe's prose were a parody of philosophy. It strikes me that in Poe's tales, the thought is being worked out that, now anyway, philosophy exists only as a parody of philosophy, or rather as something indistinguishable from the perversion of philosophy, as if to overthrow the reign of reason, the reason that philosophy was born to establish, is not alone the task of, let us say, poetry, but is now openly the genius or mission of philosophy itself. (Cavell, 2003, 99-100)

12 Si la littérature parodie la philosophie, si elle en subvertit le discours, elle ne s'y substitue pas, mais elle contribue à déplacer la philosophie elle-même et la force à se 
repenser, à se reconfigurer sous la forme d'une auto-parodie: il n'y aurait de philosophie que dans la perversion, le détournement, le dérèglement de la philosophie. Mais cette redécouverte de la philosophie par elle-même est le produit de sa rencontre avec la littérature. Plus exactement, elle est le fruit de son installation provisoire mais décisive dans l'espace littéraire, du différend qui en a résulté et de la réconciliation paradoxale de ces deux modes d'écriture et de pensée, rapport conflictuel que Cavell décrit littéralement comme une scène de ménage : "as if literature in America were forgiving philosophy, not without punishing it, for having thought that it could live only in the banishing of literature.» (Cavell, 2003, 108) Si l'on file la métaphore consciemment mélodramatique de Cavell, littérature et philosophie en Amérique ne sauraient vivre longtemps l'une sans l'autre et ne seraient nulle part ailleurs plus chez elles que dans les textes d'Emerson et de Poe. Et pour Cavell, ce n'est pas un hasard si Emerson et Poe, mais aussi Hawthorne et Thoreau, justement parce qu'ils sont américains, s'efforcent d'imaginer une manière d'habiter le monde et de vivre avec autrui : "all undertake to imagine domestication, or inhabitation - as well, being Americans, they might. » (Cavell, 2003, 108) Rien d'étonnant non plus à ce que Cavell suggère, à la fin de son essai, que le mariage et les querelles de ménage plus ou moins violentes qui le ponctuent sont les lieux privilégiés où vient se réfléchir l'ensemble de ces préoccupations philosophiques et littéraires, mais surtout nationales. Dans cette perspective, la parodie de la philosophie qui préside à la première version de «The Philosophy of Furniture " peut se lire comme l'une de ces scènes où littérature et philosophie se cherchent querelle pour mieux pervertir de concert le jargon de la raison spéculative et lui substituer une pensée de l'ordinaire, en l'occurrence de l'ameublement dans l'Amérique du XIX siècle.

\section{Critique de l'ameublement}

13 Reste toutefois à penser ce que cette philosophie dit, non seulement de ces meubles dont elle a fait son affaire, mais aussi de l'Amérique et peut-être de la philosophie ellemême, au moins dans son rapport à la littérature. La philosophie de l'ameublement selon Poe est d'abord une critique et une sémiotique avant d'être une esthétique. Le texte se divise en deux parties de longueur inégale : dans la première, Poe entreprend, on l'a dit, de dénoncer le mauvais goût de ses contemporains en matière d'ameublement, tandis que dans la seconde, il décrit, en guise de contrepoint, une pièce à l'aménagement jugé parfait, dont le propriétaire s'est assoupi sur l'un des deux sofas que compte le lieu. Dans la version de 1845, il établit d'emblée que l'ameublement est un révélateur du goût et du caractère d'une nation : interroger le choix des meubles et leur agencement dans l'espace, c'est en somme faire œuvre de philosophie sociale. S'il loue les Anglais pour la justesse de leurs choix, il reproche aux Écossais un tempérament trop peu artistique et, à la faveur d'un double jeu de mots, se moque des Hollandais aussi bien que des Espagnols qui selon lui ne sauraient, ni les uns ni les autres mais pour des raisons différentes, reconnaître la véritable utilité des rideaux: "The Dutch have, perhaps, an indeterminate idea that a curtain is not a cabbage. In Spain they are all curtains - a nation of hangmen ${ }^{10}$." Mais à l'en croire, seuls les Américains n'ont rien compris à rien et font tout à l'envers: "The Yankees alone are preposterous. " La critique porte sur le penchant quasi atavique de ses contemporains à l'exhibition spectaculaire, à la surexposition serait-on tenté de dire en ces temps d'invention du daguerréotype (1839), et suit un double mouvement : Poe commence par 
mettre en lumière le triste spectacle de l'argent-roi avant de s'attacher aux conséquences sociales et politiques des nouvelles modalités techniques de la vision qui s'inventent au XIX ${ }^{\mathrm{e}}$ siècle. Anticipant la Théorie de la classe de loisir de Thorstein Veblen (1899), il offre une analyse au vitriol de la consommation ostentatoire que pratiquent ses concitoyens dans le but d'asseoir leur "distinction" et rapporte notamment le besoin compulsif de faire étalage de sa richesse à l'absence, aux États-Unis, d'un ordre social fondé sur le sang et l'hérédité :

We have no aristocracy of blood, and having therefore as a natural, and indeed as an inevitable thing, fashioned for ourselves an aristocracy of dollars, the display of wealth has here to take the place and perform the office of the heraldic display in monarchical countries. By a transition readily understood, and which might have been as readily foreseen, we have been brought to merge in simple show our notions of taste itself. [...] [In] America the coins current being the sole arms of the aristocracy, their display may be said, in general, to be the sole means of the aristocratic distinction [...].

Dans l'espace égalitaire de la démocratie, l'aspiration au luxe est monnaie courante et le statut, fonction non de la naissance mais de la fortune. Celui-ci se mesure même exclusivement à la capacité à engager des dépenses somptuaires dont le montant confirme la place de l'individu qui s'y adonne sur l'échelle sociale. En ce sens, l'intérêt du meuble ne tient plus tant à son utilité pratique ou décorative qu'à son prix. La valeur d'échange de l'objet supplante sa valeur d'usage ou plutôt se confond avec elle et fait la preuve (test) du capital social de son propriétaire, à défaut de garantir la qualité de ses jugements de goût (taste) :

In short, the cost of an article of furniture has at length come to be, with us, nearly the sole test of its merit in a decorative point of view - and this test, once established, has led the way to many analogous errors, readily traceable to the one primitive folly. [...] The corruption of taste is a portion or a pendant of the dollarmanufacture.

Jouant sur la paronomase entre test et taste, Poe montre que le critère de la distinction en Amérique n'est pas esthétique ou culturel, mais financier. Il faut exposer, exhiber sa richesse pour exister, car c'est le seul moyen d'obtenir la reconnaissance tant attendue. L'ostentation est le fruit des « institutions républicaines » de l'Amérique, terreau d'un capitalisme débridé : elle a pour effet de mettre les citoyens en concurrence les uns avec les autres, de pousser chacun à toujours plus de débauche consumériste dans le vain espoir de remporter une course effrénée mais sans fin. La philosophie de l'ameublement débouche donc sur une critique des rapports économiques qui régissent la société américaine et Poe apparaît du même coup en précurseur de Marx aussi bien que de Veblen. Elle s'apparente également à une sémiotique avant l'heure, car les meubles y sont compris comme des symboles au sens de Peirce, ce que Poe appelle des "pendants ", c'est-à-dire des signes qui réfèrent à un objet en vertu d'un rapport arbitrairement convenu. Ce sont même des signes de signes, puisqu'ils renvoient d'abord à leur prix, c'est-à-dire au signe de la richesse que constitue la monnaie, le dollar désormais réifié et fétichisé (Hayes, 2002, 115), et ce fétichisme de la monnaie désigne à son tour toute l'étendue du déclin esthétique de la nation.

Cette mise au jour des enjeux de la consommation ostentatoire et de la société du spectacle qu'elle produit se conjugue à une réflexion sur les nouvelles formes de visibilité et les nouvelles modalités de la vision qui naissent au XIXe siècle. À suivre Poe, le désir d'exposer ses précieuses possessions au regard envieux d'autrui conduit en effet à les placer sous un éclairage toujours plus cru, comme pour en accroître l'éclat : 
" Glare is a leading error in the philosophy of American household decoration - an error easily recognized as deduced from the perversion of taste just specified. We are violently enamoured of gas and glass. » Révélateur de la vulgarité américaine avant tout, l'éclairage au gaz démultiplié par l'emploi d'abat-jour en verre taillé (« cut glass shade ») et la présence excessive de miroirs dans toutes les pièces («the exaggerated employment of mirrors ») crée une lumière criarde de fort mauvais goût, comme l'atteste le lexique de Poe pour caractériser cette brillance artificielle et tape-à-l'œil ("flashiness ", " gaudy abominations»). Mais surtout, de gaudy à gas, glass, glare et glitter se construit progressivement une chaine allitérative où la reprise insistante du phonème / gl / rappelle discrètement le nom de Hegel, dont Derrida a fait dans Glas le signifiant par excellence de la métaphysique (Derrida, 1974). Effacé de la version de 1845, Hegel demeure pourtant dans la lettre du texte et sa présence spectrale signe une nouvelle fois, toujours sous rature, la condamnation de la métaphysique, implicitement associée à la dégradation du caractère national qui s'effectue sous la double et pernicieuse influence du capitalisme et de la modernité technologique.

17 C'est toutefois sous le nom d'un autre de ses représentants que la philosophie subit explicitement les attaques de Poe: Jeremy Bentham. Évoquant les tapis tout en couleurs qui meublent trop souvent les demeures des masses vulgaires (« the dwelling of the rabble »), Poe en fait des inventions diaboliques qui concentrent l'ensemble des faisceaux de sa critique :

these are but the wicked invention of a race of time-servers and money-lovers children of Baal and worshippers of Mammon - Benthams, who to spare thought and economize fancy, first cruelly invented the Kaleidoscope, and then established joint-stock companies to twirl it by steam.

Le rejet du kaléidoscope, que Jonathan Crary compte au nombre des nouveaux modes de rapport au visible qui voient le jour au XIXe siècle (Crary, 1992), doit être mis en relation avec la critique des miroirs qui court dans le texte. Ajoutés à la mention du nom de Bentham, ces deux éléments suggèrent la dénonciation d'une société panoptique où la visibilité absolue de tout et de tous est le meilleur moyen d'assurer le contrôle de chacun. Et le rapprochement avec l'actionnariat capitaliste vient souligner la complicité de l'économique, du politique et du philosophique dans l'instauration de ces technologies de la surveillance (Sherman, 1981, 31).

Au terme de la première partie du texte, une fois passée au révélateur de la philosophie de l'ameublement, c'est donc une Amérique surexposée que Poe nous donne à lire et à voir, une Amérique où la consommation ostentatoire, le développement de l'éclairage au gaz et la prolifération des surfaces réfléchissantes concourent à un excès de visibilité qui a pour effet d'annuler la distinction entre sphères publiques et privées. Plus exactement, tout relève désormais du public. L'intimité disparait sous les projecteurs de la modernité et cette abolition du privé apparaît comme le double résultat d'une concurrence et d'un contrôle social accrus entre les individus. Kevin J. Hayes a récemment fait observer que cette réflexion sur les conditions de la modernité américaine avait pu être inspirée à Poe par l'observation d'une pratique immobilière propre à la ville de New York dont «The Philosophy of Furniture » pourrait bien être un commentaire à l'oblique (Hayes, 2002, 103-107). À l'époque, New York manque en effet de logements pour accueillir une population de plus en plus nombreuse et les prix des loyers s'en ressentent cruellement, ce qui occasionne de fréquents déménagements dans l'espoir de trouver moins cher ailleurs. Tout au long du XIX siècle, le $1^{\text {er }}$ mai est justement connu à New York sous le nom de «Moving Day»: ce jour-là, les baux 
immobiliers arrivent à échéance et chaque année, toujours plus de locataires envahissent les rues à la recherche de conditions de vie meilleures dans d'autres immeubles et d'autres quartiers. Dès les années 1820, le sud de Manhattan est la scène d'un désordre sans précédent qui conduit les autorités de la ville à suspendre l'activité économique pour la journée et l'on peut voir des milliers de gens transporter leurs meubles et leurs effets personnels sur des carrioles surchargées, en quête d'un logement plus approprié à leur niveau de ressources (Erickson, 1995, 778-779; Burrow et Wallace, 1999, 479). Si Poe est encore à Philadelphie lorsque "The Philosophy of Furniture " paraît pour la première fois, il est à New York en 1845. L'année précédente, le 14 mai 1844, il remarque déjà, dans l'une de ses contributions au Columbia Spy:

We are not yet over the bustle of the first May. "Keep Moving" have been the watchwords for the last fortnight. The man who, in New York, should be so bold as not to peregrinate on the first, would, beyond doubt, attain immortality as "The Great unmoved" - a title applied by Horne, the author of Orion, to one of his heroes Akinetos, the type of the spirit of Apathy. (Poe, 1929, 29)

Par ailleurs, les quatre fois que «The Philosophy of Furniture » paraît en revue, dans Burton's et le Broadway Journal on l'a dit, mais aussi dans The Spirit of the Times (1840) et le Weekly Mirror (1845), c'est au mois de mai, comme si Poe entendait que sa philosophie de l'ameublement fût d'abord lue dans le contexte de « Moving Day » auquel il ne fait pourtant allusion que de biais. À la fois homme de son temps et à l'écart de son époque, Poe est bien un « contemporain » au sens paradoxal qu'Agamben confère à ce mot dans son commentaire des Considérations inactuelles de Nietzsche :

Celui qui appartient véritablement à son temps, le vrai contemporain, est celui qui ne coïncide pas parfaitement avec lui ni n'adhère à ses prétentions, et se définit, en ce sens, comme inactuel ; mais précisément pour cette raison, précisément par cet écart et cet anachronisme, il est plus apte que les autres à percevoir et à saisir son temps. (Agamben, 2009, 24)

21 Car ce qui se donne à voir au moment de "Moving Day", à la faveur de ce déménagement généralisé, de ce remue-ménage qui agite tout New York, c'est précisément l'excès de visibilité, l'exposition de l'intimité domestique aux yeux de tous que Poe décèle également dans les principes qu'adoptent ses contemporains en matière d'aménagement intérieur, mais dont ils ne perçoivent ni le sens, ni les dangers.

\section{«Figures of no meaning »}

Le spectacle d'une invraisemblable désorganisation publique tous les $1^{\text {er }}$ mai peut aussi expliquer pourquoi Poe insiste tant sur les motifs de l'arrangement, de l'ordonnancement, de l'ajustement, en un mot, de la composition harmonieuse (keeping), terme qu'il emprunte au lexique de la peinture, mais qui s'applique évidemment, de manière réflexive, à son propre texte :

We speak of the keeping of a room as we would of the keeping of a picture - for both the picture and the room are amenable to those undeviating principles which regulate all varieties of art; and very nearly the same laws by which we decide on the higher merits of a painting, suffice for decision on the adjustment of a chamber.

Face aux mouvements désordonnés de New-Yorkais que l'on imagine pris de panique et cherchant désespérément à se loger, il décrit au contraire, dans la seconde partie du texte, une pièce parfaitement ordonnée, où règne le calme de la nuit : « Repose speaks in all ${ }^{11}$.» Tout s'y oppose à ce qu'il vient d'évoquer et de dénoncer, de sorte que cette 
chambre paraît " tout de luxe discret, de douce intimité, d'ordre et de sévère beauté ", " toute tournée sur elle-même, comme repue " (Justin, 1991, 164 et 178): les couleurs sont sobres et se répondent harmonieusement pour plonger la chambre dans une atmosphère pourprée; les arabesques du papier-peint font écho à celles du tapis ; sur les murs, les tableaux alternent entre représentations paysagères et portraits de femmes, tandis qu'un seul miroir est accroché, mais il est positionné de telle sorte qu'il ne risque de refléter aucun des occupants de la pièce à moins de se tenir juste devant lui.

On a souvent vu dans cette perfection quasi immobile, ce calme rassurant, un contrepoint aux contes de terreur où chambres et salons sont des lieux d'angoisse et de mort. Si « The Philosophy of Furniture » peut se lire dans la continuité de ce qu'Henri Justin a justement appelé «la série des chambres », car il en reprend et en concentre les principaux motifs architecturaux et ornementaux, il s'en distingue néanmoins en ce qu'il propose une structure dramatique minimale (un seul personnage endormi, si l'on excepte le narrateur-voyeur qui fait intrusion dans la pièce et grâce à qui le lecteur fait le tour des lieux sans toutefois perturber le sommeil de leur propriétaire) au sein de laquelle «[les] tensions s'équilibrent, la dialectique est apaisée » (Justin, 1991, 187). Même si la plupart du temps, "The Philosophy of Furniture » est compté au nombre des contes de Poe, il existerait donc une dichotomie entre la fiction et l'essai et, partant, entre littérature et philosophie: d'un côté, des tensions exacerbées, toujours en mouvement, qui ne trouvent leur résolution que dans la mort et de l'autre, une synthèse hiératique et finalement inoffensive. Pour beaucoup, cette dichotomie repose notamment sur le rôle dévolu à l'arabesque : là où, dans « Ligeia » par exemple, l'espace s'anime et les involutions des arabesques manifestent la plongée terrifiante de la psyché " dans le champ du vertige $»^{12}$, les motifs qui ornent le tapis et le papier-peint dans "The Philosophy of Furniture " restent figés. Comme le rappelle la première partie du texte : « whether on carpets, or curtains, or tapestry, or ottoman-coverings, all upholstery of this nature should be rigidly Arabesque.» Si le conseil se veut impératif et si la philosophie de l'ameublement suppose de la rigueur, comment ne pas entendre, dans l'emploi de l'adverbe rigidly, l'interruption du mouvement, l'annulation des forces qui s'exercent sur l'âme dont le tapis est précisément la figure ("The soul of the apartment is the carpet ", dit Poe dans le même passage), l'arrêt du tourbillon de la folie et l'apaisement de l'esprit, ou du moins l'espoir d'un tel apaisement? Des contes à la philosophie, l'arabesque se rigidifie et deviendrait, non plus le signe du chaos terrifiant qui saisit la psyché, mais celui de l'ordre et du confort mental aussi bien que social.

Observant la double valence de l'arabesque chez Poe dans « Domestic Terror and Poe's Arabesque Interior ", Jacob Berman remet toutefois en cause le partage générique que cette oscillation semble recouper et montre que, dans les deux cas, l'arabesque est la figure d'une altérité fantasmée que le sujet incorpore à son espace domestique et entreprend en vain de garder à distance et sous contrôle en l'esthétisant sous la forme d'un motif abstrait. Reflet de la fascination qu'exerce un orient de fiction sur l'imaginaire littéraire et populaire américain au XIX ${ }^{\mathrm{e}}$ siècle, la fortune de l'arabesque manifeste également la peur de l'étranger qu'il s'agit de juguler pour préserver l'homogénéité, voire la pureté, du sujet et de la communauté. Si «The Philosophy of Furniture » est exemplaire de ce double effort d'incorporation et de maitrise de l'altérité, il est également, et comme dans les contes, exemplaire de son échec. Loin de 
demeurer rigide et stable, l'arabesque s'y avère en effet un puissant opérateur de déstabilisation :

The arabesque destabilizes the sanctity of home with [...] its proximity to the grotesque, primitive, and monstrous. The choice of European furnishings which occupy Poe's paradigmatic American apartment are accompanied by "arabesque devices" and a landscape painting of "the fairy grottoes of Stanfield". Thus, the threat of domestic terror is pregnant in Poe's scene of domestic tranquility, for the arabesque and grotesque potency of Poe's "devices" and "grottoes" is latent within the meditative calm which this cosmopolitan elements help to conjure. (Berman, 2005, 137)

Or, dans la mesure où l'espace intérieur chez Poe est littéralement "psychédélique ", au sens où «il manifeste la psyché » comme le rappelle avec force Henri Justin dans Avec Poe jusqu'au bout de la prose (Justin, 2009, 105) ${ }^{13}$, ce que révèle la présence de l'arabesque au cœur même de l'appartement, c'est aussi et surtout la présence terrifiante de l'autre en soi et même, plus exactement, la reconnaissance de soi-même comme un autre. Comme le montre Anthony Vidler dans The Architectural Uncanny, l'expérience de l'étrangeté de/dans l'espace domestique opère également au niveau psychologique :

[The uncanny's] favorite motif was precisely the contrast between a secure and homely interior and the fearful invasion of an alien presence; on a psychological level, its play was one of doubling, where the other is, strangely enough, experienced as a replica of the self, all the more fearsome because apparently the same. (Vidler, 1992, 3)

On comprend dès lors que le seul miroir qui se trouve dans la pièce ne renvoie pas le reflet de l'occupant assoupi sur le sofa : « it is hung so that a reflection of the person can be obtained from it in none of the ordinary sitting-places of the room. » Positionné de la sorte, le miroir n'offre pas au sujet l'image rassurante de lui-même, ni ne confirme son identité, car celle-ci n'a rien de stable. S'il reflète quoi que ce soit, ce sont d'abord les arabesques qui ornent les murs, ces arabesques qui ne représentent rien d'identifiable ou de déterminé ( « cycloid figures of no meaning ») et qui sont, de ce point de vue, des figures de la différence. Ce qui apparaît dans le miroir et ce que donne à voir "The Philosophy of Furniture », c'est l'image vide d'un soi absent à lui-même qui refuse d'ouvrir les yeux de peur de ne pas se reconnaitre. En fin de compte, la philosophie de l'ameublement s'apparente donc à une mimésis non-mimétique où la projection de soi dans l'espace domestique ne ramène pas à l'identité, mais ouvre sur le vertige de la différence ${ }^{14}$. Et si, dans "The Philosophy of Furniture», la philosophie s'invite en littérature et admet ne pas avoir de lieu propre sur la scène américaine, la littérature de son côté n'accueille pas tant la philosophie chez elle qu'elle ne reconnaît à son tour sa propre vicariance.

\section{BIBLIOGRAPHIE}

AGAMBEN, Giorgio, Nudités, traduit de l'italien par Martin Rueff, Paris, Rivages, 2009. 
AMFREVILLE, Marc, « Poétique du spectral dans The Scarlet Letter de N. Hawthorne », Hawthorne et la pensée du roman, Philippe Jaworski, dir., Paris, Michel Houdiard, 2006, 45-60.

BENJAMIN, Walter, Paris, Capitale du XIX ${ }^{e}$ siècle. Le livre des Passages, traduit de l'allemand par Jean Lacoste, Paris, Les Editions du Cerf, 1989.

BERMAN, Jacob Rama, « Domestic Terror and Poe's Arabesque Interior », ESC : English Studies in Canada, 31, n 1 (March 2005), 128-50.

BURROW, Edwin G., et Mike WALLACE, Gotham: A History of New York City to 1898, New York, Oxford University Press, 1999.

CAVELL, Stanley, The Senses of Walden, Expanded Edition, Chicago, University of Chicago Press, 1992 [1981].

---, « Being Odd, Getting Even (Descartes, Emerson, Poe) », dans Emerson's Transcendental Etudes, David Justin Hodge, éd., Stanford, Stanford University Press, 2003, 83-109.

CAZÉ, Antoine, « Reflet et réflexion dans The Scarlet Letter », Hawthorne et la pensée du roman, Philippe Jaworski, dir., Paris, Michel Houdiard, 2006, 11-22.

COOPER, James Fenimore, The Pathfinder (1840), William P. Kelly, dir., Oxford, Oxford University Press, 1999 [1992].

CRARY, Jonathan, Techniques of the Observer : On Vision and Modernity in the Nineteenth Century, Cambridge, MIT Press, 1992.

DAVENPORT, Guy, The Geography of the Imagination : Forty Essays, San Francisco, North Point, 1981. DERAIL-IMBERT, Agnès, « La Philosophie à la plage », Études anglaises 59, nº 3 (2006), 304-18. DERRIDA, Jacques, Glas, Paris, Galilée, 1974.

DERRIDA, Jacques et Anne DUFOURMANTELLE, De l'hospitalité, Paris, Calmann-Lévy, 1997.

DESCARTES, René, Méditations métaphysiques, Paris, GF-Flammarion, 1992 [1979].

DICKENS, Charles, The Life and Adventures of Martin Chuzzlewit (1843), Patricia Ingham, éd., Londres, Penguin Classics, 2004.

EMERSON, Ralph Waldo, Essays and Lectures, Joel Porte, éd., New York, The Library of America, 1983.

ERICKSON Alana J., " Moving Day », dans The Encyclopedia of New York, New Haven, Yale University Press, 1995, 778-79.

FLEMING, Paul, « Dream Chamber : Poe, Baudelaire, Benjamin », Zeitschrift für deutsche Philologie, $\mathrm{n}^{\circ} 4,2010,579-92$.

GORDON, Rae Beth, «Interior Decoration in Poe and Gilman », Literature, Interpretation, Theory 3, 1991, 85-99.

GROS, Frédéric, Marcher : une philosophie, Paris, Flammarion, 2011 [2009].

HAWTHORNE, Nathaniel, The Scarlet Letter and Other Writings, Leland S. Person, éd., New York, Norton Critical Edition, 2005.

HAYES, Kevin J., « The Flaneur in the Parlor : Poe's "Philosophy of Furniture" ", Prospects 27, 2002, 103-19.

JUSTIN, Henri, Poe dans le champ du vertige, Paris, Klincksieck, 1991.

---, Avec Poe jusqu'au bout de la prose, Paris, Gallimard, 2009. 
KINGWELL, Mark, « Tables, Chairs, and Other Machines for Thinking », Practical Judgments: Essays in Culture, Politics and Interpretation, Toronto, University of Toronto Press, 2002, 229-47.

LACAN, Jacques, « Le séminaire sur “La Lettre volée” », Écrits I, Paris, Le Seuil, 1999 [1966].

MAUPASSANT, Guy de, Pour Gustave Flaubert, préface de Maurice Nadeau, Paris, Editions complexes, 1986.

NIETZSCHE, Friedrich, Crépuscule des idoles, Paris, Gallimard, « Folio », 1995 [1974].

POE, Edgar Allan, « The Philosophy of Furniture », Edgar Allan Poe : Poetry and Tales, Patrick F. Quinn, éd., New York, The Library of America, 1984, 382-87.

---, « The Philosophy of Furniture », The Collected Works of Edgar Allan Poe, vol. 2: Tales \& Sketches I, T. O. Mabbott, éd., Cambridge, The Belknap Press of Harvard University Press, 1978, 494-504.

--, « The Philosophy of Furniture », Burton's Gentleman's Magazine, mai 1840, 243-45, en ligne, http://www.eapoe.org/works/essays/philfurn.htm (page consultée le 5 avril 2013).

---, « House Furniture », Broadway Journal, 3 mai 1845, 1, 273-75, en ligne, http://www.eapoe.org/ works/essays/philfrnb.htm (page consultée le 5 avril 2013).

- -, « Philosophie de l'ameublement », traduit de l'anglais par Charles Baudelaire, CEuvres en prose, Paris, Gallimard, « La Pléiade », 1951, 971-78.

---, « Bon-Bon », Edgar Allan Poe : Poetry and Tales, Patrick F. Quinn, éd., New York, The Library of America, 1984, 164-80.

---, « Ligeia », Edgar Allan Poe : Poetry and Tales, Patrick F. Quinn, éd., New York, The Library of America, 1984, 262-77.

---, « Mellonta Tauta », Edgar Allan Poe : Poetry and Tales, Patrick F. Quinn, éd., New York, The Library of America, 1984, 871-85.

---, Marginalia, dans Edgar Allan Poe : Essays and Reviews, G. R. Thompson, éd., New York, The Library of America, 1984.

---, Doings of Gotham, Jacob E. Spannuth et T. O. Mabbott, éd., Pottsville, PA, Jacob E. Spannuth, 1929.

POPE, Alexander, «The Second Epistle of the Second Book of Horace Imitated », The Poems of Alexander Pope, John Butt, éd., Londres, Methuen \& Co, 1963, 650-58.

PLATON, La République, Paris, GF-Flammarion, 1995 [1966].

RICHARD, Claude, Edgar Allan Poe journaliste et critique, Paris, Klincksieck, 1974.

---, « Poe et l'esthétique du double », Le Double dans le romantisme anglo-américain, Christian La Cassagnère, dir., Clermont-Ferrand, Association des publications de la faculté de Lettres et Sciences humaines, 1984, 155-64.

SHERMAN, G. W., « Poe and the Panopticon », Poe Studies 14, December 1981, 31, en ligne, http:// www.eapoe.org/pstudies/ps1980/p1981204.htm (page consultée le 5 avril 2013).

SMITH, Patricia C., « Poe’s Arabesque », Poe Studies 7, n² 2, December 1974, 42-45.

THOREAU, Henry David, A Week on the Concord and Merrimack Rivers, Walden, The Maine Woods, Cape Cod, Robert R. Sayre, éd., New York, The Library of America, 1985.

---, « Walking », Collected Essays and Poems, Elizabeth Hall Witherell, éd., New York, The Library of America, 2001, 225-55. 
---, Cap Cod, traduit de l'anglais par Pierre-Yves Pétillon, Paris, Imprimerie nationale, 2000.

VIDLER, Anthony, The Architectural Uncanny : Essays in the Modern Unhomely, Cambridge, MIT Press, 1992.

\section{NOTES}

1. Dans The Senses of Walden, Cavell parle de l'ouvrage de Thoreau, paru en 1854, comme du produit d'une époque « pré-philosophique » de la culture américaine, un temps où la philosophie ne se distingue pas encore de la littérature, de la théologie, de la politique et de l'économie (Cavell, 1992, xiii-xiv). Au XVIII ${ }^{\mathrm{e}}$ siècle déjà, Cotton Mather et Jonathan Edwards, à qui l'on doit respectivement The Christian Philosopher (1721) et Freedom of Will (1754), illustrent cette intrication sur le versant théologique, tandis que les écrits des Pères fondateurs l'attestent sur le versant politique, et sans doute pourrait-on observer dès A Modell of Christian Charity de John Winthrop (1630) cet entremêlement de la philosophie à d'autres discours, théologiques et politiques en l'occurrence.

2. Dans De l'hospitalité, Derrida relève, à la suite de Benveniste, la parenté étymologique qui unit l'hôte et l'ennemi (hostis) et forge le néologisme " hostipitalité " pour décrire ce qui se joue dans la venue et l'accueil d'un étranger chez soi (Derrida et Dufourmantelle, 1997, 45).

3. Je remercie Bruno Monfort, fin connaisseur de Poe et déterreur de trésors littéraires, d'avoir attiré mon attention sur ce texte.

Hasards du calendrier, j'écris ce texte alors que l'U.F.R. d'études anglophones de l'université Paris Diderot vient de quitter son lieu historique, rue Charles V dans le Marais, pour s'installer dans le bâtiment Olympe de Gouges, rejoignant ainsi le campus de l'université sur le site Paris Rive Gauche. Dans le même temps a vu le jour à Paris Diderot un «Pôle philosophie » qui, rappelant la décision de l'université de ne pas se doter d'une U.F.R ou d'un département de philosophie lors de sa création, entend néanmoins constituer un lieu virtuel de rassemblement pour l'ensemble des philosophes disséminés dans les différentes composantes. Son inauguration a été marquée, le 8 mars 2013, par une journée d'études intitulée «La Philosophie en ménages » dont l'ambition était de réfléchir à ce que signifie pratiquer la philosophie hors les murs, à la croisée d'autres disciplines et depuis leur demeure institutionnelle. À sa manière, cette étude se veut un écho à ce questionnement dont elle partage le fonds métaphorique et conceptuel.

4. L'édition du Webster de 1828 donne la définition suivante: «When applied to any particular department of knowledge, it denotes the collection of general laws or principles under which all the subordinate phenomena or facts relating to that subject, are comprehended " (en ligne, http://1828.mshaffer.com/d/search/word,philosophy, page consultée le 5 avril 2013). L'emploi du mot "philosophy » en ce sens traduit aussi l'« attachement [de Poe] à la pensée du siècle des lumières » (Richard, 1974, 292).

5. Si Baudelaire parlera à ce propos de la "genèse d'un poème » et de la "méthode » de sa composition, il n'en reste pas moins que ces deux "philosophies » sont liées en ceci qu'il y est à chaque fois question de la composition conjointe d'un lieu et d'un texte et de la recherche d'un effet.

6. Dans Marcher : une philosophie, Frédéric Gros retrace quelques-unes des étapes de cette tradition et médite sur le rapport du mouvement et de la pensée, de la marche et de la philosophie (Gros, 2011).

7. Voir à ce sujet l'étude d'Agnès Derail-Imbert intitulée «La Philosophie à la plage » (DerailImbert, 2006). Dans The Geography of the Imagination, Guy Davenport rappelle que l'année de la première publication de "The Philosophy of Furniture " est aussi l'année où James Fenimore Cooper, alors au faîte de sa renommée, fait paraître The Pathfinder, manière de suggérer que Poe 
est doublement en décalage par rapport à la littérature et à la philosophie telles qu'elles s'inventent et se pratiquent alors aux Etats-Unis et qui font des grands espaces leur terrain d'élection (Davenport, 1981, 5).

8. Le mot est rapporté par Maupassant dans un texte intitulé «Étude sur Gustave Flaubert » et paru les 19 et 26 janvier 1884 dans la Revue Bleue avant d'être reproduit la même année en tête des Lettres de Gustave Flaubert à Georges Sand (Maupassant, 1986, 38).

9. Le texte de «The Philosophy of Furniture » étant assez bref et facilement accessible dans la version de 1840 comme dans celle de 1845 sur le site de The Edgar Allan Poe Society of Baltimore (voir http://www.eapoe.org/works/info/petpf.htm\#text03; page consultée le 4 avril 2013), il nous a semblé inutile d'indiquer le numéro de page pour chacune des citations. On trouvera, dans la liste des ouvrages cités, les références des principales éditions.

10. Si le jeu sur hangmen se comprend aisément, l'emploi de cabbage appelle peut-être davantage d'explications : derrière l'allusion à un légume qui serait particulièrement prisé des Hollandais, il faut entendre le sens qu'a le mot chez les tailleurs où il désigne une rognure d'étoffe.

11. Paul Fleming a fait observer que dans repose, s'entend aussi le nom de Poe: si les meubles parlent la langue du repos, c'est aussi Poe qui s'exprime silencieusement à travers eux (Fleming, 2010, 584).

12. Citons ce passage bien connu de «Ligeia » où Poe décrit la chambre nuptiale imaginée par le narrateur: "The material was the richest cloth of gold. It was spotted all over, at irregular intervals, with arabesque figures, about a foot in diameter, and wrought upon the cloth in patterns of the most jetty black. But these figures partook of the true character of the arabesque only when regarded from a single point of view. By a contrivance now common, and indeed traceable to a very remote period of antiquity, they were made changeable in aspect. To one entering the room, they bore the appearance of simple monstrosities; but upon a farther advance, this appearance gradually departed; and step by step, as the visitor moved his station in the chamber, he saw himself surrounded by an endless succession of the ghastly forms which belong to the superstition of the Norman, or arise in the guilty slumbers of the monk. The phantasmagoric effect was vastly heightened by the artificial introduction of a strong continual current of wind behind the draperies - giving a hideous and uneasy animation to the whole.» (Poe, 1984a, 271, nous soulignons) L'étude de Rae Gordon, «Interior Decoration in Poe and Gilman », met l'accent sur le lien entre l'arabesque, la folie et le mouvement de l'écriture dans "Ligeia " et «Berenice » (Gordon, 1991, 85-93), tandis que dans « Poe's Arabesque », Patricia Smith montre que, dans les contes, l'arabesque a le plus souvent partie liée à un mouvement de dissolution et l'arrivée imminente de la mort (Smith, 1974, 45).

13. Ce n'est pas un hasard si c'est justement à ce moment de sa lecture, lorsqu'il évoque une nouvelle fois « la série des chambres » après l'analyse menée dans Poe dans le champ du vertige, que Justin convoque «The Philosophie of Furniture » : "L'art de Poe est toujours "psychédélique" au sens étymologique du terme : il manifeste la psyché. Mettant en scène une conscience, il la situe par rapport à cette scène psychique qui pour chacun de nous est le lieu véritable. On s'expliquerait mal autrement que Poe ait prôné l'unité de lieu tout en se démarquant des classiques, qu'il ait écrit une "Philosophie de l'ameublement", qu'il ait posé dans le premier récit policier un problème de chambre close, qu'il ait composé une série de contes paysages, que les enceintes abondent dans toute son œuvre, que la question de l'univers, enfin, soit ramenée dans Eurêka à celle de la saisie conceptuelle de l'espace psychocosmique. » (Justin, 2009, 105-106)

14. De ce point de vue, «The Philosophy of Furniture » doit être rapproché des nombreux contes où Poe procède à une critique en règle de ce que Claude Richard a appelé «l'esthétique du double ", qu'il s'agisse de "Ligieia » et de "The Fall of the House of Usher », mais aussi de «Eleonora », « Morella », «William Wilson» et bien sûr "The Oval Portrait ». Dans "Poe et l'esthétique du double », s'appuyant sur la lecture « disséminale » de « The Purloined Letter » que propose Derrida dans La Carte postale, Richard montre en effet que «le monde de la narration 
poesque est d'abord le lieu où le miroir confronte un autre miroir et y voit les lignes de fuite de la distorsion spéculaire ». Là où narrateurs et personnages cherchent à toute force à rabattre le double sur le même " pour écarter la pire menace d'un double plus terrible ", le récit fait vaciller les correspondances et fonctionne au contraire comme un opérateur de différence (Richard, 1984, 159 et 164).

Cette image d'un miroir qui ne reflète pas ce qui lui fait face, mais ouvre l'espace incertain de la pensée, entre littérature et philosophie, est également exploitée par Hawthorne dans «The Custom House » : "On Hester Prynne's story, therefore, I bestowed much thought. [...] My imagination was a tarnished mirror. It would not reflect, or only with miserable dimness, the figures with which I did my best to people it. » (Hawthorne, 2005, 28). Marc Amfreville et Antoine Cazé ont mis en lumière, chacun à sa manière, la dimension spéculative de cette "poétique du spectral » (Amfreville, 2006) où le motif du miroir reflète le vacillement identitaire autant qu'il permet de le penser (Cazé, 2006).

\section{RÉSUMÉS}

À suivre Stanley Cavell, l'Amérique ne se serait exprimée philosophiquement que dans sa littérature. Qu'en est-il dès lors de ce ménage entre littérature et philosophie qui s'établit en Amérique? Que signifie pour la philosophie habiter en littérature et pour la littérature être l'hôte de la philosophie? Qu'est-ce que s'installer dans la maison d'autrui ? Qu'est-ce qu'emménager? Avec quels meubles? Quels sont les meubles de la philosophie ? Et y aurait-il une philosophie du meuble? Ces questions trouvent un écho singulier, et peut-être quelques réponses, dans un court texte d'Edgar Poe justement intitulé "The Philosophy of Furniture » (1840). Si Poe n'est pas un philosophe, ses textes donnent assurément à penser et sa philosophie de l'ameublement est une invitation à réfléchir à l'état d'une Amérique saisie par la passion du matérialisme, à ce que nos meubles disent de notre rapport à nous-mêmes et aux autres, mais aussi à ce lien paradoxal qui unit littérature et philosophie.

According to Stanley Cavell, America has "never ever expressed itself philosophically," unless it be "in the metaphysical riot of its greatest literature." What is this odd coupling, this strange union of literature and philosophy? What does it mean for philosophy to move in with literature? What does it mean to set up house in someone else's home? With what furniture? Does philosophy have its own furniture? Is there a philosophy of furniture? These questions find a surprising echo, and maybe a few answers, in a short text by Edgar Allan Poe precisely entitled "The Philosophy of Furniture" (1840). Poe may not be a philosopher, but his texts surely are food for thought and his philosophy of furniture is an invitation to reflect on the materialist craze that drives nineteenth-century America, but also to think about what our furniture reveal of our relationship to ourselves and to others, and to consider the paradoxical link that unites philosophy and literature.

\section{INDEX}

Mots-clés : Edgar Allan Poe, Stanley Cavell, philosophie, littérature, ameublement Keywords : Edgar Allan Poe, Stanley Cavell, philosophy, literature, furniture 
AUTEUR

THOMAS CONSTANTINESCO

Université Paris Diderot 